\title{
Comparison of Laparoscopic Enclosed Electromechanical Morcellation and Vaginal Enclosed Scalpel Morcellation at Laparoscopic Myomectomy: A Prospective Randomized Trial
}

\author{
Ali Akdemir ${ }^{1}$, Sabahattin Ari ${ }^{2}$, Enes Taylan ${ }^{3}$, Firat ÖKMEN ${ }^{4}$, and Cagdas Sahin ${ }^{1}$ \\ ${ }^{1}$ Ege University \\ ${ }^{2}$ Bakircay University \\ ${ }^{3}$ Yale University \\ ${ }^{4}$ Affiliation not available
}

March 2, 2022

\begin{abstract}
Objective: To compare the laparoscopic enclosed electromechanical morcellation (LEM) with vaginal enclosed scalpel morcellation (VSM) in laparoscopic myomectomy procedures. Design: Prospective randomized interventional clinical study. Setting: Tertiary university hospital. Population or Sample: One hundred eighteen patients who underwent laparoscopic myomectomy. Methods: All myomectomy procedures were performed laparoscopically. After myomectomy, tissue removal was accomplished via either LEM using the previously described in-glove morcellation technique or VSM. In the VSM group, myoma was placed into an endoscopic bag and removed through the vagina via posterior colpotomy after scalpel morcellation. Main Outcome Measures: Primary outcome: tissue removal time. Secondary outcome: rescue analgesia requirement, postoperative Visual Analog Scale (VAS) scores, postoperative 3th month scores of Female Sexual Function Index (FSFI). Results: The median tissue removal time was longer in the LEM group (25 min (Range: 14-55)) than the VSM group (20 min (Range: 6-38) ( $\mathrm{p}=.001)$. Rescue analgesia requirement was significantly higher in the LEM group than the VSM group (mean rank: 56.92 vs 40.92 doses, respectively; $\mathrm{p}<.001)$. There was no significant difference between the groups at postoperative VAS scores. There was no significant difference between preoperative and postoperative 3th month total scores of FSFI and subdomains in the LEM group. Conversely, all subdomains and total scores of FSFI (26.5 (16.7-34.8) vs. 22.7 (15.2-28.7) except pain significantly worsened three months after operation in the VSM group. Conclusion: LEM was associated with a longer tissue removal time and increased postoperative analgesic requirement. On the other hand, VSM was associated with worsened postoperative sexual function from baseline.
\end{abstract}

\section{Hosted file}

Manuscript.docx available at https://authorea.com/users/463219/articles/558370-comparisonof-laparoscopic-enclosed-electromechanical-morcellation-and-vaginal-enclosed-scalpelmorcellation-at-laparoscopic-myomectomy-a-prospective-randomized-trial

\section{Hosted file}

Table 1.docx available at https://authorea.com/users/463219/articles/558370-comparisonof-laparoscopic-enclosed-electromechanical-morcellation-and-vaginal-enclosed-scalpelmorcellation-at-laparoscopic-myomectomy-a-prospective-randomized-trial

\section{Hosted file}


Table 2.docx available at https://authorea.com/users/463219/articles/558370-comparisonof-laparoscopic-enclosed-electromechanical-morcellation-and-vaginal-enclosed-scalpelmorcellation-at-laparoscopic-myomectomy-a-prospective-randomized-trial

\section{Hosted file}

Table 3.docx available at https://authorea.com/users/463219/articles/558370-comparisonof-laparoscopic-enclosed-electromechanical-morcellation-and-vaginal-enclosed-scalpelmorcellation-at-laparoscopic-myomectomy-a-prospective-randomized-trial

\section{Hosted file}

Table 4.docx available at https://authorea.com/users/463219/articles/558370-comparisonof-laparoscopic-enclosed-electromechanical-morcellation-and-vaginal-enclosed-scalpelmorcellation-at-laparoscopic-myomectomy-a-prospective-randomized-trial

\section{Hosted file}

Table 5.docx available at https://authorea.com/users/463219/articles/558370-comparisonof-laparoscopic-enclosed-electromechanical-morcellation-and-vaginal-enclosed-scalpelmorcellation-at-laparoscopic-myomectomy-a-prospective-randomized-trial

\section{Hosted file}

Figure 1.docx available at https://authorea.com/users/463219/articles/558370-comparisonof-laparoscopic-enclosed-electromechanical-morcellation-and-vaginal-enclosed-scalpelmorcellation-at-laparoscopic-myomectomy-a-prospective-randomized-trial

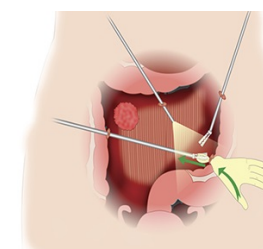

A

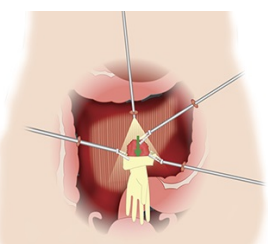

B

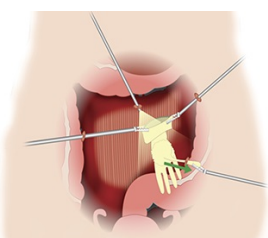

C

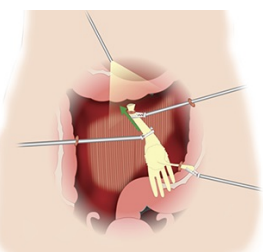

D

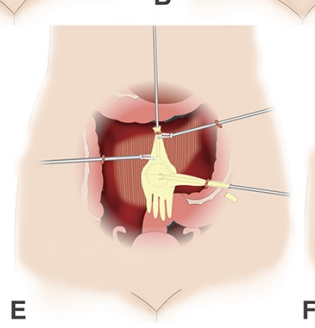

F

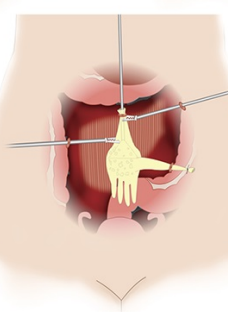

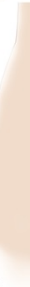

G

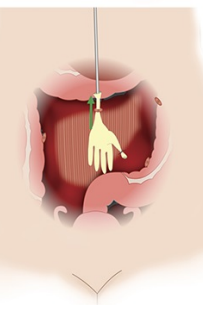

